\title{
Critical exponent of quantum phase transitions driven by colored noise
}

\author{
D. Nagy and P. Domokos \\ Institute for Solid State Physics and Optics, Wigner Research Centre, \\ Hungarian Academy of Sciences, H-1525 Budapest P.O. Box 49, Hungary
}

\begin{abstract}
We demonstrate that criticality in a driven-dissipative system is strongly influenced by the spectral properties of the reservoir. We study the open-system realization of the Dicke model, where a bosonic cavity mode couples to a large spin formed by two motional modes of an atomic Bose-Einstein condensate. The cavity mode is driven by a high frequency laser and it decays to a Markovian bath, while the atomic mode interacts with a colored reservoir. We reveal that the soft mode fails to describe the characteristics of the criticality. We calculate the critical exponent of the superradiant phase transition and identify an inherent relation to the low-frequency spectral density function of the colored bath. We show that a finite temperature of the coloured reservoir does not modify qualitatively this dependence on the spectral density function.
\end{abstract}

PACS numbers: 05.30.Rt,42.50.Pq,37.10.Vz,03.75.Kk

\section{INTRODUCTION}

Quantum phase transitions in driven-dissipative systems opened up a novel research area in the field of critical phenomena [1-5]. These transitions lie beyond the standard classification of classical dynamical or equilibrium phase transitions, and define completely new universality classes [6-9]. In an open quantum system, the critical behaviour appears in the state formed by the dynamical equilibrium of the external driving and dissipation processes. The abrupt symmetry breaking change of such a steady state takes place when the external control parameters are continuously tuned across the critical point [10-13]. The correlation functions at the critical point are determined by nonequilibrium noise rather than thermal or ground-state quantum fluctuations [14-18].

Recent cavity quantum electrodynamics (cavity QED) experiments with ultracold atoms strongly coupled to the radiation field of a high-finesse resonator led to the observation of a variety of quantum critical phenomena [19-28]. Owing to the high degree of precision in controlling the system parameters in these experiments, the critical regime can be explored with sufficient resolution, such that quantitative measurements of the critical exponent are available [29]. Remarkably, the theoretical description of the system can be significantly simplified to treating the dynamics of only a few relevant modes of the interacting many-body system. Therefore, manyatom cavity QED systems offer a suitable platform to describe generic features of quantum criticality.

The effective Hamiltonian underlying the observed superradiant phase transition in Refs. [19, 24] is closely related to the Dicke-model which describes the interaction of a single bosonic mode with a large spin- $\mathrm{N}$ degree of freedom. In the thermodynamic limit, $N \rightarrow \infty$, the Dicke-model is exactly solved by the corresponding mean-field model [30]. Approaching the critical point, the incoherent population in the bosonic mode diverges following a power law with exponent $1 / 2$ [31]. Dissipation can be taken into account in the mean-field theory which amounts to a model of coupled boson modes with lin- earized fluctuations. This is still a solvable model which results in a critical exponent 1 [32]. The experiment, which is intrinsically a dissipative system due to the presence of photon losses from the cavity, confirmed that the value of exponent is indeed in the vicinity of 1 [29]. It was argued that the dissipative system, although the actual temperature is $T=0$, is a classical system and the critical point corresponds to a thermal phase transition at a certain effective temperature [33]. The exponent 1 observed experimentally is in accordance with this claim. On the other hand, one may note that different bosonic modes are subject to reservoirs at different effective temperatures, i.e., there is no global effective temperature. Eluding the classical analogy, too, the bosonic modes exhibit a certain amount of entanglement even in the lossy system. Moreover, the logarithmic negativity, used as an entanglement measure, undergoes a non-analytic behaviour in the critical point [32].

In a recent letter, we studied how the characteristics of the dissipation determine the critical exponent [34]. We introduced a simple model based on two coupled boson modes which corresponds well to the open-system Dicke model describing effectively the relevant dynamics of the experimental system in the normal phase, i.e., below the critical point. One of the modes representing the cavity mode is lossy simply due to the photon leakage from the cavity, which is a Markovian process to very high accuracy. Besides this simple relaxation process, we took into account that the other bosonic mode representing the density-wave mode of the ultracold atom cloud relevant to the criticality is also damped. The principal loss mechanism is the collision induced scattering into the continuum of density-wave excitation modes. The damping process is a kind of Beliaev damping of phonons dressed by photons $[35,36]$. It is a complicated theoretical task to describe this effect at a microscopic level. For simplicity, we modelled the dissipation of the atomic boson mode phenomenologically, by assuming that it is subjected to a colored reservoir with sub-ohmic spectral density function. Within this approach, we pointed out the existence of a relation between the critical exponent 
and the exponent of the power-law spectral density function of the reservoir. As a consequence, the critical exponent can take on, in principle, any value in an interval, including values below 1 . In the present paper we generalize our calculation into two important directions. First, we extend the class of sub-ohmic reservoirs to the superohmic ones, i.e., we treat in full generality the spectral density function. Second, we assume finite temperature of this reservoir in order to check if the unusual exponent values survive thermal effects at finite temperature.

The paper is structured as follows. In Sect. 2, we recall the main steps to derive the effective two-boson model used for studying the dynamics in the vicinity of the critical point. We define the colored reservoir on a microscopic basis, and introduce the Keldysh path integral of the action for the lossy bosonic modes. In Sect. 3, we consider the critical phenomenon by using the various components of the Keldysh Green's function. First, we discuss the singularities of the retarded Green's function, and then we study the spectral properties of both the photonic and the atomic modes by means of the Keldysh component of the corresponding reduced Green's functions. The critical exponent is extracted numerically from the divergence of the correlation functions. The finite temperature effects are calculated within the same formalism in Sect. 4. Finally, we conclude in Sect. 5.

\section{MODEL}

\section{A. The Dicke Hamiltonian and its bosonization}

Ultracold atom experiments where a laser-driven BoseEinstein condensate is dispersively coupled to a highfinesse optical cavity realize the driven Dicke model [31], that is

$$
H=\omega_{a} a^{\dagger} a+\omega_{b} \hat{S}_{z}+y\left(a e^{i \omega_{p} t}+a^{\dagger} e^{-i \omega_{p} t}\right) \frac{\hat{S}_{x}}{\sqrt{N}} .
$$

The large spin of $N / 2$ represents the motional state of $N$ atoms distributed in two different motional modes of the ultracold gas [31]. The laser pump incident on the atoms is far detuned from an atomic transition, while it is quasi-resonant with a single cavity mode. Hence, the atoms mediate effective photon scattering between the laser and the cavity mode, that is accompanied by atomic recoil. This scattering process gives rise to the time dependent interaction term in Eq. (1) [37].

In a frame rotating at the driving frequency $\omega_{p}$, the Hamiltonian becomes time independent,

$$
H=\delta_{a} a^{\dagger} a+\omega_{b} \hat{S}_{z}+y\left(a+a^{\dagger}\right) \frac{\hat{S}_{x}}{\sqrt{N}},
$$

where the detuning parameter $\delta_{a}=\omega_{a}-\omega_{p}$ expresses the effective photon energy in the harmonically driven system. Since the spin represents the collective motional state of the atoms, the corresponding frequency given by the recoil frequency $\left(\omega_{b}=\omega_{R}\right)$ is the smallest frequency scale in the system $\left(\omega_{R} / \omega_{p} \leq 10^{-10}\right)$.

The superradiant phase transition takes place in the thermodynamic limit $(N \rightarrow \infty)$ of the Dicke model [38]. When reaching a critical coupling $y_{c}^{\mathrm{GS}}=\sqrt{\delta_{a} \omega_{b}}$, a coherent photon field amplitude builds up spontaneously in the cavity and the spin becomes polarized along the $S_{x}$ direction. Due to the global coupling of the model (all the spins are coupled to the same bosonic mode, i.e., the coordination number is infinite) the phase transition can be described within mean-field theory, which is exact in the thermodynamic limit [39]. In order to develop the meanfield theory, one introduces the Holstein-Primakoff boson representation of the collective spin $\hat{S}_{-}=\sqrt{N-b^{\dagger} b} b$, $\hat{S}_{+}=b^{\dagger} \sqrt{N-b^{\dagger} b}$ and $\hat{S}_{z}=b^{\dagger} b-N / 2$. The Hilbert space of the boson operator $b$ is truncated for $b^{\dagger} b \geq N$. Then the operators are displaced by their mean values $a \rightarrow \alpha+a, b \rightarrow \beta+b$ and the square roots in the Hamiltonian are expanded up to second order in the operators $a, b$, and their adjoints (the corrections are of the order of $1 / N)$. The mean fields are readily solved in Ref. [40] and with dissipation in Ref. [32]. In the normal phase $\alpha=\beta=0$, while in the superradiant phase the mean fields are nonzero: $\alpha, \beta \propto \sqrt{N}$.

Our central goal is to study the dissipative effects on the quantum critical point of the model. We can freely choose to approach the critical point of the system from the side of the normal phase $\left(y<y_{c}\right)$, where the Dicke problem reduces to the following simple Hamiltonian of two coupled bosonic modes,

$$
H_{S} / \hbar=\delta_{a} a^{\dagger} a+\omega_{b} b^{\dagger} b+\frac{y}{2}\left(a+a^{\dagger}\right)\left(b+b^{\dagger}\right) .
$$

We shall consider the interplay between two dissipation channels. Mode $a$ is assumed to be a cavity mode driven by a laser and emitting "high frequency" photons into the vacuum, thus its decay is unaffected by the interaction between modes $a$ and $b$. Mode $b$ couples, on the other hand, to a colored reservoir that is sampled at the eigenfrequencies of the coupled system described by the above Hamiltonian. We apply the Keldysh path integral approach to calculate the dissipative effects of the two reservoirs [33], which is a suitable tool to obtain directly the steady-state properties of the system.

\section{B. Markovian dissipation of the cavity mode}

For mode $a$ the photon loss dissipation can be described in frequency space by the action

$$
S_{a}=\int \frac{\mathrm{d} \omega}{2 \pi}\left(a_{\mathrm{cl}}^{*}, a_{\mathrm{q}}^{*}\right)\left(\begin{array}{cc}
0 & \omega-\delta_{a}-i \kappa \\
\omega-\delta_{a}+i \kappa & 2 i \kappa
\end{array}\right)\left(\begin{array}{c}
a_{\mathrm{cl}} \\
a_{\mathrm{q}}
\end{array}\right),
$$

where $a_{\mathrm{cl}}(\omega)$ and $a_{\mathrm{q}}(\omega)$ are the classical and quantum fields that correspond to the operator $a$. Action (4) describes the standard Markovian decay process of the cavity mode by means of a constant $\kappa$. The high-frequency 
drive ensures that the cavity dissipation is unaffected by the interaction between modes $a$ and $b$, since $y \ll \omega_{p}$ even in the superradiant phase. The parameter $\delta_{a}=\omega_{a}-\omega_{p}$ expresses the fact that the cavity mode frequency is referenced to the driving frequency $\omega_{p}$.

\section{General description of the dissipation of the atomic mode}

Let us consider a bosonic bath composed of modes $c_{k}$ interacting with mode $b$ according to the Hamiltonian

$$
H_{b c}=\sum_{k} \omega_{k} c_{k}^{\dagger} c_{k}+\sum_{k} g_{k}\left(b^{\dagger} c_{k}+c_{k}^{\dagger} b\right) .
$$

Integrating out the reservoir modes $c_{k}$ in the Keldysh path integral formalism, we obtain the following dissipative action for the oscillator $b$,

$$
S_{b}=\int_{\omega}\left(b_{\mathrm{cl}}^{*}, b_{\mathrm{q}}^{*}\right)\left(\begin{array}{cc}
0 & \omega-\omega_{b}-K^{A} \\
\omega-\omega_{b}-K^{R} & D
\end{array}\right)\left(\begin{array}{l}
b_{\mathrm{cl}} \\
b_{\mathrm{q}}
\end{array}\right),
$$

where $b_{\mathrm{cl}}(\omega)$ and $b_{\mathrm{q}}(\omega)$ are the classical and quantum fields corresponding to mode $b$, with self energies

$$
K^{R / A}(\omega)=\sum_{k} \frac{g_{k}^{2}}{\omega-\omega_{k} \pm i \eta}
$$

that incorporate the frequency shift and damping originating from the coupling to the reservoir. Note that this is sometimes referred to as the level-shift function in quantum optics [41, 42]. Finally, the Keldysh component of the action is

$$
D(\omega)=2 \pi i \sum_{k} g_{k}^{2} F(\omega) \delta\left(\omega-\omega_{k}\right),
$$

with the function $F(\omega)=\operatorname{coth}\left\{(\hbar \omega-\mu) / k_{B} T\right\}$, which takes into account the thermal occupation of the bath modes at a given frequency. For generality, we enable a chemical potential $\mu$ for the reservoir which is needed for the low temperature limit of the bosonic modes of the reservoir. We consider the thermal effects only in mode $b$, since the high-frequency mode $a$ is still effectively at zero temperature, $\hbar \omega_{b} \sim k_{B} T \ll \hbar \omega_{p}$.

\section{Colored reservoir}

The genuine ingredient of the system is that the reservoir coupled to the low-frequency mode $b$ is colored. Such a non-Markovian bath arises from quasi-particle scattering in the Bose-Einstein condensate, e.g., from a Beliaevtype damping process $[35,36]$. Instead of providing a microscopic theory, we consider a general class of reservoirs described by the coupling-density profile

$$
\rho(\omega)=\sum_{k} g_{k}^{2} \delta\left(\omega-\omega_{k}\right)
$$

The essential point is that this bath is sampled at the eigenfrequencies of the coupled system. Since we focus on the soft-mode criticality of the phase transition, the relevant property of the bath is its low-frequency behaviour. We use the classification of the colored reservoirs introduced by Leggett [43], and we assume a power-law frequency dependence of $\rho(\omega)$ with exponent $0<s<2$,

$$
\rho(\omega)=\gamma \Theta(\omega) \frac{\left(\omega / \omega_{b}\right)^{s}}{1+\left(\omega / \omega_{M}\right)^{4}}
$$

where $\Theta(\omega)$ is the Heaviside function, $\gamma$ is the dissipation strength and $\omega_{M}$ is a cutoff frequency. The cutoff at the fourth power in the denominator enables us to consider both the sub-Ohmic $(s<1)$ and the super-Ohmic $(s>1)$ cases. Our results will be, of course, independent of the the cutoff in the end. The range $s>2$ can be easily described in the same way and gives no new physics.

The coupling-density function determines completely the level-shift function Eq. (7a), which can be expressed by using complex analytic extension. When $\rho(\omega)$ is an analytic function of the real variable $\omega$, the level-shift function can be extended to the complex plane analytically

$$
K(z)=\int_{0}^{\infty} \frac{\rho(\omega)}{z-\omega} \mathrm{d} \omega,
$$

such that the retarded $K^{R}(\omega)$ is extended analytically to the upper half plane $(\operatorname{Im} z>0)$, and the advanced $K^{A}(\omega)$ is extended to the lower half plane $(\operatorname{Im} z<0)$. The reverse expression reads

$$
K^{R / A}(\omega)=\lim _{\eta \rightarrow 0} K(\omega \pm i \eta) .
$$

To uncover the properties of the complex function $K(z)$, we evoke the relation

$$
\lim _{\eta \rightarrow 0} \frac{1}{x \pm i \eta}=\mathcal{P} \frac{1}{x} \mp i \pi \delta(x) .
$$

Substituting it into the integral in Eq. (10), we obtain

$$
\lim _{\eta \rightarrow 0} K(\omega \pm i \eta)=\mathcal{P} \int_{0}^{\infty} \frac{\rho\left(\omega^{\prime}\right)}{\omega-\omega^{\prime}} \mathrm{d} \omega^{\prime} \mp i \pi \rho(\omega),
$$

where the real part describes the frequency shift and the imaginary part yields the damping rate. As $\rho(\omega)$ is nonzero only for $\omega>0, K(z)$ has a branch cut along the positive real axis, and its imaginary part vanishes along the negative real axis. At this point our investigation differs from the standard textbook example, where $K^{R / A}(\omega)$ is evaluated at a large positive frequency. Usually, in the frame of the Markov approximation, the lower boundary of the integral in Eq. (10) is extended to $-\infty$, which would continue the branch cut of $K(z)$ to the whole real axis. On the contrary, we are looking for the effects of dissipation around zero frequency, hence we continue analytically $K^{R / A}(\omega)$ to the complex plane by keeping the lower limit of the integral. 


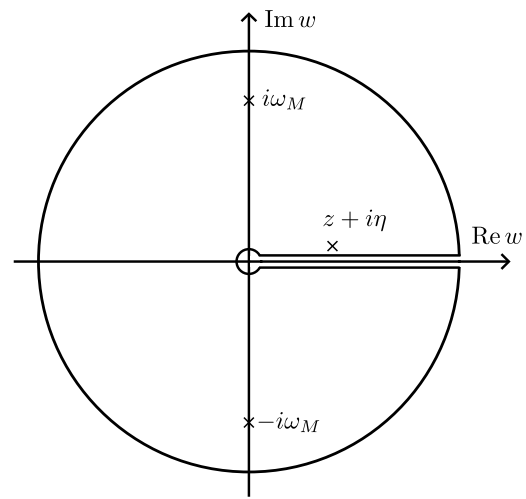

FIG. 1: Contour for calculating the integral. It starts in the infinitesimal vicinity of the origin and goes along the real axis, then makes a full circle in the infinity. It comes back along the real axis, to be finally closed by the circle surrounding the origin, where the fractional power function is non-analytic. The symbol $\times$ indicates the poles.

With the coupling-density profile given in Eq. (9), it is possible to calculate $K^{R}(z)$ by evaluating the integral

$$
K^{R}(z)=\int_{0}^{\infty} \frac{\rho(\omega)}{z-\omega+i \eta} \mathrm{d} \omega
$$

by complex contour integration. First, we extend the function of $\omega$ in the integrand analytically to the complex plane. Since $K^{R}(z)$ has a branch cut along the positive real axis, we choose the complex function $(-z)^{s}$ with $s<1$, and calculate the following integral on the closed contour given in Fig. 1,

$$
R(z)=\oint_{C} \frac{\left(-w / \omega_{b}\right)^{s}}{(z-w+i \eta)\left[\left(w / \omega_{M}\right)^{2}+1\right]} \mathrm{d} w
$$

where $z$ and $w$ are complex numbers. Note that we assume $s<1$ for simplicity, when $s>1$ one should separate the fractional part of $s$ in the numerator as $\left(-w / \omega_{b}\right)^{[s]}\left(-w / \omega_{b}\right)^{\{s\}}$, and recombine these functions after the integration. The radius of the small circle around $w=0$ is finally made to approach zero, while the radius of the large circle is taken to infinity, that eliminates their contributions to the integral. The two integrals performed in opposite directions along the positive real axis give different results, since they are on opposite sides of the branch cut: above and below the positive real axis, the phase of the function $(-w)^{s}$ is $e^{-i s \pi}$ and $e^{i s \pi}$, respectively. After exchanging the limits of the integral that goes on the line below the branch cut, we obtain

$$
R(z)=K^{R}(z)\left[e^{-i s \pi}-e^{i s \pi}\right] / \gamma .
$$

On the other hand, the contour integral Eq. (15) can be calculated by means of the residue theorem. The integrand has three poles at $w=z+i \eta$ and $w= \pm i \omega_{M}$. All of them are inside the contour, resulting

$$
R(z)=2 \pi i\left[-\frac{\left(-(z+i \eta) / \omega_{b}\right)^{s}}{\left(\frac{z+i \eta}{\omega_{M}}\right)^{2}+1}+\operatorname{Re}\left\{\frac{\left(i \omega_{M} / \omega_{b}\right)^{s}}{1-i \frac{z+i \eta}{\omega_{M}}}\right\}\right]
$$

The second term increases with the cutoff frequency $\omega_{M}$, and it gives a large contribution to the real part of $K^{R}(z)$, hence renormalizing the frequency $\omega_{b}$. To eliminate the effects of the cutoff, we do the standard renormalization by taking the limit $\omega_{M} \rightarrow \infty$ and incorporating the diverging real part into $\omega_{b}$. In this way, the first term results

$$
R(z)=-2 \pi i\left(-\frac{z+i \eta}{\omega_{b}}\right)^{s} .
$$

Substituting this result into the left hand side of Eq. (16), we obtain

$$
K^{R}(z)=\frac{\pi \gamma}{\sin s \pi}\left(-\frac{z+i \eta}{\omega_{b}}\right)^{s} .
$$

We keep in in the expression to mark that the calculation is not valid when the pole of the integrand is on the positive real axis. Normally, when calculating the level-shift function for real $\omega$, it is the $\pm i \eta$ which distinguishes between the retarded and advanced Green's functions having the symmetry $K^{A}(\omega)=\left[K^{R}(\omega)\right]^{*}$. Here, in the $\eta \rightarrow 0$ limit, we obtain

$$
K^{R / A}(\omega)=\frac{\gamma \pi}{\sin s \pi}\left(\omega / \omega_{b}\right)^{s}\left[\Theta(\omega) e^{\mp i s \pi}+\Theta(-\omega)\right] .
$$

The Heaviside-function $\Theta(\omega)$ assures the property stemming from Eq. (13) that the level-shift function is real for $\omega<0$. The above result is valid up to $s<c$, where $c=2$ is the exponent of the cutoff. Note that the trigonometric factors are periodic in $s$, namely the $K^{R}(\omega>0)$ part is invariant to $s \rightarrow s+1$, and $K^{R}(\omega<0)$ is invariant to $s \rightarrow s+2$.

In order to complete the calculation of the elements of the action in Eq. (6), we note that the Keldysh component Eq. (7b) is simply expressed as

$$
D(\omega)=2 i \pi \rho(\omega) .
$$

\section{NON-EQUILIBRIUM PHASE TRANSITION}

The driven Dicke model exhibits a non-equilibrium phase transition that we analyzed previously in Ref. [32] without the colored bath. We demonstrated that the cavity photon loss fundamentally changes the critical properties of the system with respect to the closed Hamiltonian model. The second order phase transition takes place in the steady state, where the critical exponent is changed to 1 (instead of the mean-field exponent $1 / 2$ found in the ground state). Also, the critical point is shifted upward by the cavity decay rate. In the following, we describe how the colored reservoir modifies the critical point of the open system. 


\section{A. The full Keldysh action}

The Keldysh action corresponding to the interaction term in the Hamiltonian, in Eq. (3), reads

$S_{a b}=-\frac{y}{2} \int_{\omega}\left[\left(a_{q}+a_{q}^{*}\right)\left(b_{c l}+b_{c l}^{*}\right)+\left(a_{c l}+a_{c l}^{*}\right)\left(b_{q}+b_{q}^{*}\right)\right]$,

expressed with the classical and quantum components. The counterrotating terms in the Hamiltonian results in terms like $a_{c l} b_{q}$ or $a_{q}^{*} b_{c l}^{*}$. Hence, in order to cast the Keldysh action into the standard matrix form, one doubles the variable space by introducing fields with negative frequency

$$
\mathbf{v}(\omega)=\left(\begin{array}{c}
a_{c l}(\omega) \\
a_{c l}^{*}(-\omega) \\
b_{c l}(\omega) \\
b_{c l}^{*}(-\omega) \\
a_{q}(\omega) \\
a_{q}^{*}(-\omega) \\
b_{q}(\omega) \\
b_{q}^{*}(-\omega)
\end{array}\right)
$$

Then the total Keldysh action

$$
S=S_{a}+S_{b}+S_{a b}
$$

can be written as

$$
S=\frac{1}{2} \int \frac{\mathrm{d} \omega}{2 \pi} \mathbf{v}^{\dagger}\left(\begin{array}{cc}
0 & {\left[\mathbf{G}_{4 \times 4}^{A}\right]^{-1}(\omega)} \\
{\left[\mathbf{G}_{4 \times 4}^{R}\right]^{-1}(\omega)} & \mathbf{D}_{4 \times 4}^{K}(\omega)
\end{array}\right) \mathbf{v} .
$$

The $4 \times 4$ blocks are matrix Green's functions:

$$
\begin{gathered}
{\left[\mathbf{G}_{4 \times 4}^{R}\right]^{-1}(\omega)=} \\
\left(\begin{array}{cccc}
{\left[G_{a}^{R}\right]^{-1}(\omega)} & 0 & -y / 2 & -y / 2 \\
0 & {\left[G_{a}^{A}\right]^{-1}(-\omega)} & -y / 2 & -y / 2 \\
-y / 2 & -y / 2 & {\left[G_{b}^{R}\right]^{-1}(\omega)} & 0 \\
-y / 2 & -y / 2 & 0 & {\left[G_{b}^{A}\right]^{-1}(-\omega)}
\end{array}\right)
\end{gathered}
$$

with the inverse retarded Green's functions of each mode

$$
\begin{gathered}
{\left[G_{a}^{R}\right]^{-1}(\omega)=\omega-\delta_{a}+i \kappa,} \\
{\left[G_{b}^{R}\right]^{-1}(\omega)=\omega-\omega_{b}-K^{R}(\omega) .}
\end{gathered}
$$

Note that the advanced and retarded Green's functions are hermitian adjoint, $\left[G_{4 \times 4}^{A}\right]^{-1}(\omega)=\left[\left[G_{4 \times 4}^{R}\right]^{-1}(\omega)\right]^{\dagger}$, whereas the scalars $\left[G^{A}\right]^{-1}(\omega)=\left[\left[G^{R}\right]^{-1}(\omega)\right]^{*}$ are complex conjugate. The inverse of the Keldysh component describing the fluctuations is a diagonal matrix

$$
\mathbf{D}_{4 \times 4}^{K}(\omega)=\operatorname{diag}(2 i \kappa, 2 i \kappa, D(\omega), D(-\omega)),
$$

whith $D(\omega)$ is given in Eq. (21).

\section{B. The soft-mode pole}

Second order quantum criticality is accompanied by the vanishing of the frequency of one of the excitation modes, i.e., the soft mode. In a dissipative Markovian system the soft-mode frequency is complex, and the critical properties are encoded in its imaginary part, the socalled adiabatic decay rate [44]. Although this is not the case when the system is non-Markovian, we first calculate the characteristic frequencies of the system. These are identified by the poles of the retarded Green's function continued analytically to the lower half of the complex plane. Note, however, that one cannot attribute a single complex frequency to describe the dynamics of the soft mode because it does not correspond simply to a Lorentzian resonance. Moreover, the non-Markovian character of the system is crucially enhanced close to the critical point.

Let us continue analytically the level shift function to the second Riemann sheet

$$
K_{\mathrm{II}}^{R}(z)=\gamma \frac{\pi e^{-i s \pi}}{\sin s \pi}\left(z / \omega_{0}\right)^{s}
$$

then use the symmetry property $K_{\mathrm{II}}^{A}(z)=\left[K_{\mathrm{II}}^{R}(z)\right]^{*}$, and finally replace the argument $-\omega$ of the advanced components with $-z^{*}$, which ensures the convergence of the Fourier integrals. The poles are defined by the characteristic equation $\operatorname{det}\left[\mathbf{G}_{4 \times 4}^{R}\right]^{-1}(z)=0$, i.e.,

$$
\begin{array}{r}
{\left[(z+i \kappa)^{2}-\delta_{a}^{2}\right]\left[(z-i \Gamma(z))^{2}-\left(\omega_{b}+\Delta(z)\right)^{2}\right]} \\
-y^{2} \delta_{a}\left(\omega_{b}+\Delta(z)\right)=0
\end{array}
$$

where $\Gamma(z)=\left(K_{\mathrm{II}}^{R}(z)-K_{\mathrm{II}}^{A}\left(-z^{*}\right)\right) /(2 i)$ and $\Delta(z)=$ $\left(K_{\mathrm{II}}^{R}(z)+K_{\mathrm{II}}^{A}\left(-z^{*}\right)\right) / 2$.

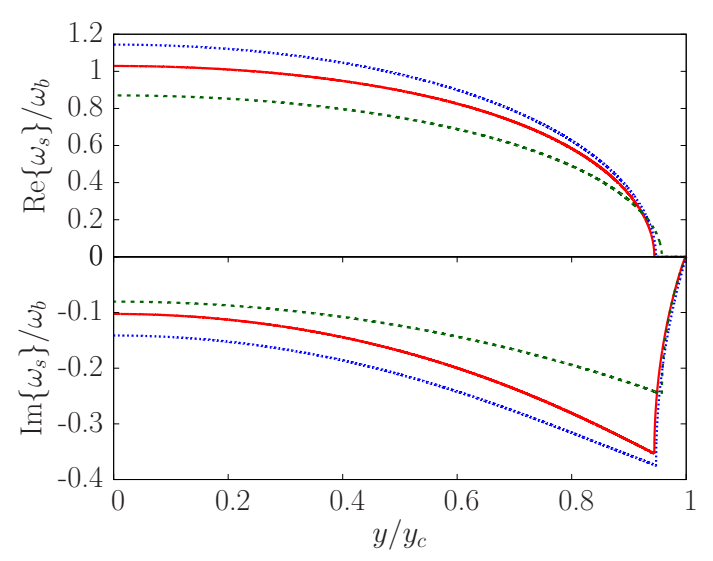

FIG. 2: (Color online) Real and imaginary parts of the softmode frequency as a function of the coupling $y$. The parameters are $s=0.4$ (solid red) 0.8 (dashed green), 1.2 (dotted blue), $\mu=0, \gamma=0.1 \omega_{b}$ and $\delta_{a}=\kappa=2 \omega_{b}$.

Due to the colored reservoir the 'decay' $\Gamma(z)$ and the 'frequency shift' $\Delta(z)$ depend explicitly on the complex frequency. As a result Eq. (30) becomes an implicit equation for the characteristic frequencies. According to the 
symmetry property $K_{\mathrm{II}}^{A}(z)=\left[K_{\mathrm{II}}^{R}(z)\right]^{*}$, the poles come in pairs, if $z_{1}$ is a pole, then $z_{2}=-z_{1}^{*}$ is also a pole of the retarded photon Green's function. This means that the poles are purely imaginary or they come in pairs with opposite real part but the same imaginary part.

Fig. 2 shows how the soft mode frequency solution of Eq. (30) vanishes at the critical point as a function of the control parameter $y$. We plot the soft mode for three different bath exponents $s=0.4$ (solid red), 0.8 (dashed green) and 1.2 (dotted blue). At $y=0$ and for $\delta_{a}>\omega_{b}$ the soft mode corresponds to the bare mode $b$, hence the real and imaginary parts show the frequency shift and decay of mode $b$ due to the interaction with the colored reservoir. As increasing the coupling parameter $y$, the dressing by photons leads to mode softening until reaching a threshold coupling (around $y \approx 0.95 y_{c}$ ). At this point, a linewidth bifurcation takes place [45]. We plot only the upper branch of the imaginary part, because this is the one associated with to the soft mode. The critical point is that where the imaginary part reaches zero. It proves to be independent of $\gamma$,

$$
y_{c}=\sqrt{\frac{\delta_{a}^{2}+\kappa^{2}}{\delta_{a}} \omega_{b}} .
$$

It is important to note that the colored noise dissipation does not modify the position of the critical point whereas the dissipation of mode $a$ with $\kappa$ does increase $y_{c}$. The reason is that both the decay rate and the light shift originating from the colored reservoir tend to zero at the critical point, $\Gamma(z \rightarrow 0)=0$ and $\Delta(z \rightarrow 0)=0$, where the reservoir modes are sampled at zero frequency.

\section{Correlation functions}

The soft mode pole calculated from Eq. (30) provides a good picture far from the critical point, nevertheless it fails to describe the critical dynamics and exponent. These latter can be extracted from the correlation functions which are provided for by the Keldysh component of the Green's functions.

\section{Photon correlation function}

We calculate the properties of the photon field by integrating out the low-frequency mode $b$ from the total Keldysh action in Eq. (25). This gives the photon-only action

$$
S_{a}^{\mathrm{eff}}=\int \frac{\mathrm{d} \omega}{2 \pi} \mathbf{v}_{a}^{\dagger}\left(\begin{array}{cc}
0 & {\left[\mathbf{G}_{2 \times 2}^{A}\right]^{-1}(\omega)} \\
{\left[\mathbf{G}_{2 \times 2}^{R}\right]^{-1}(\omega)} & \mathbf{D}_{2 \times 2}^{K}
\end{array}\right) \mathbf{v}_{a},
$$

where the vector $\mathbf{v}_{a}$ contains the classical and quantum components of the photon field

$$
\mathbf{v}_{a}^{\dagger}(\omega)=\left[a_{c l}^{*}(\omega), a_{c l}(-\omega), a_{q}^{*}(\omega), a_{q}(-\omega)\right]
$$

and the $2 \times 2$ photon Green's functions incorporate the effects of interaction between the modes. With the integration over mode $b$, the original inverse retarded photon Green's function transforms as $\left[\mathbf{G}_{2 \times 2, a}^{R}\right]^{-1} \rightarrow$ $\left[\mathbf{G}_{2 \times 2}^{R}\right]^{-1}=\left[\mathbf{G}_{2 \times 2, a}^{R}\right]^{-1}-\frac{y^{2}}{4} \mathbb{I}_{2 \times 2} \mathbf{G}_{2 \times 2, b}^{R} \mathbb{I}_{2 \times 2}$ with the matrix $\mathbb{I}_{2 \times 2}=\left(\begin{array}{ll}1 & 1 \\ 1 & 1\end{array}\right)$, by which we obtain

$$
\begin{aligned}
& {\left[\mathbf{G}_{2 \times 2}^{R}\right]^{-1}=} \\
& \left(\begin{array}{cc}
{\left[G_{a}^{R}\right]^{-1}(\omega)+\Sigma^{R}(\omega)} & \Sigma^{R}(\omega) \\
\Sigma^{R}(\omega) & {\left[G_{a}^{A}\right]^{-1}(-\omega)+\Sigma^{R}(\omega)}
\end{array}\right) .
\end{aligned}
$$

The photon self-energy

$\Sigma^{R}(\omega)=-\frac{y^{2}}{4}\left[\frac{1}{\omega-\omega_{b}-K^{R}(\omega)}+\frac{1}{-\omega-\omega_{b}-K^{A}(-\omega)}\right]$

has the symmetry propery $\left[\Sigma^{R}(-\omega)\right]^{*}=\Sigma^{R}(\omega)$, which we exploited in Eq. (34).

The correlation function of the cavity field can be derived from the $2 \times 2$ inverse Keldysh Green's function $\mathbf{D}_{2 \times 2}^{K}$ of the effective photon-only action (32). It is obtained similarly to the retarded photon Green's function. When integrating out the fields corresponding to mode $b$, the original inverse Keldysh component $\mathbf{D}_{2 \times 2, a}^{K}(\omega)=\operatorname{diag}(2 i \kappa, 2 i \kappa)$ transforms as $\mathbf{D}_{2 \times 2, a}^{K} \rightarrow$ $\mathbf{D}_{2 \times 2}^{K}=\mathbf{D}_{2 \times 2, a}^{K}-\frac{y^{2}}{4} \mathbb{I}_{2 \times 2} \mathbf{G}_{2 \times 2, b}^{K} \mathbb{I}_{2 \times 2}$, with the matrix $\mathbb{I}_{2 \times 2}=\left(\begin{array}{ll}1 & 1 \\ 1 & 1\end{array}\right)$. The new inverse Keldysh component is no longer diagonal,

$$
\mathbf{D}_{2 \times 2}^{K}=\left(\begin{array}{cc}
2 i \kappa+d(\omega) & d(\omega) \\
d(\omega) & 2 i \kappa+d(\omega)
\end{array}\right)
$$

with

$$
d(\omega)=-\frac{y^{2}}{4}\left[G_{b}^{K}(\omega)+G_{b}^{K}(-\omega)\right],
$$

where $G_{b}^{K}$ is the scalar Keldysh Green's function of mode $b$, that reads

$$
G_{b}^{K}(\omega)=\frac{-D(\omega)}{\left(\omega-\omega_{b}-K(\omega)\right)\left(\omega-\omega_{b}-K^{*}(\omega)\right)} .
$$

The Keldysh Green's function of the photon field is expressed from Eq. (32) as $\mathbf{G}_{2 \times 2}^{K}(\omega)=$ $-\mathbf{G}_{2 \times 2}^{R}(\omega) \mathbf{D}_{2 \times 2}^{K}(\omega) \mathbf{G}_{2 \times 2}^{A}(\omega)$, where the retarded Green's function, the inverse of Eq. (34), is

$$
\begin{aligned}
\mathbf{G}_{2 \times 2}^{R} & =\frac{1}{\operatorname{det}\left[\mathbf{G}_{2 \times 2}^{R}\right]^{-1}} \\
& \left(\begin{array}{cc}
{\left[G_{a}^{A}\right]^{-1}(-\omega)+\Sigma^{R}(\omega)} & -\Sigma^{R}(\omega) \\
-\Sigma^{R}(\omega) & {\left[G_{a}^{R}\right]^{-1}(\omega)+\Sigma^{R}(\omega)}
\end{array}\right) .
\end{aligned}
$$

The advanced Green's function is calculated from the symmetry $\mathbf{G}_{2 \times 2}^{A}=\left[\mathbf{G}_{2 \times 2}^{R}\right]^{\dagger}$, and $\mathbf{D}_{2 \times 2}^{K}$ is given by Eq. (36).

The relevant part of the Keldysh Green's function is its first diagonal element which gives the Fourier 

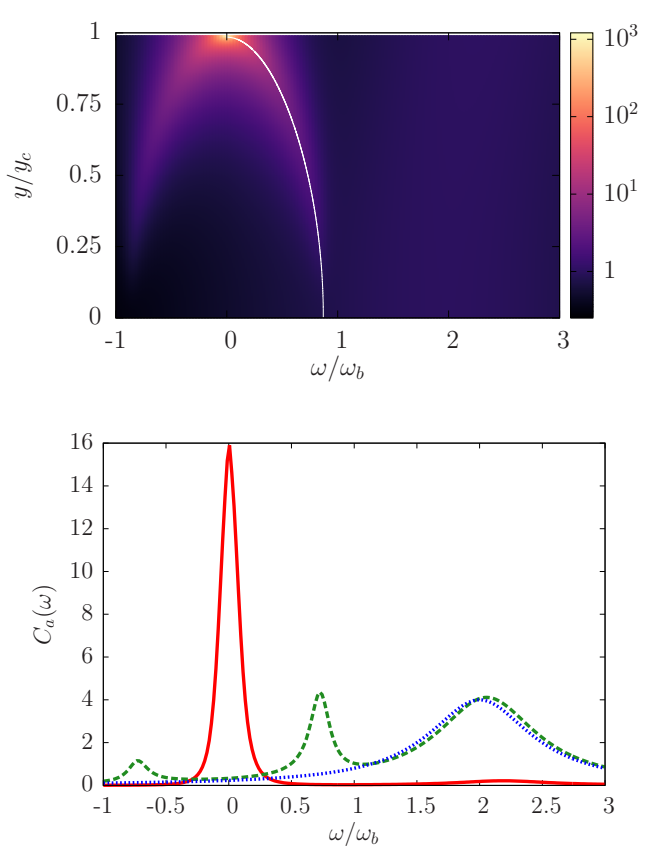

FIG. 3: (Color online) Correlation function of mode $a$ for various coupling strengths approaching the critical point. The top panel shows the correlation function in frequency space ( $\mathrm{x}-$ axis) as a function of the relative coupling strength. The white line plots the mode softening $\operatorname{Re} \omega_{s}$ shown in Fig. 2. On the bottom panel we plot the correlation function for three given values of the coulping $y=0$ (dotted blue), 0.5 (dashed green) and $0.99 y_{c}$ (solid reed). Parameters: $\delta_{a}=2 \omega_{b}, \kappa=0.5 \omega_{b}$, $\gamma=0.1, \mu=0, s=0.8$. The peaks for $y=0.99 y_{c}$ are divided by a factor of 20 .

transform of the photon correlation function $C_{a}(t)=$ $\left\langle\left\{a(t), a^{\dagger}(0)\right\}\right\rangle$, that is $C_{a}(\omega)=\left[\mathbf{G}_{2 \times 2}^{K}\right]_{11}$,

$$
\begin{array}{r}
C_{a}(\omega)=\frac{-i}{\left|\operatorname{det}\left[\mathbf{G}_{2 \times 2}^{R}\right]^{-1}\right|^{2}}\left(2 i \kappa \left[\left|\left[G_{a}^{R}\right]^{-1}(-\omega)\right|^{2}\right.\right. \\
\left.+2 \operatorname{Re}\left\{\Sigma^{R}(\omega)\left[G_{a}^{R}\right]^{-1}(-\omega)\right\}+2\left|\Sigma^{R}(\omega)\right|^{2}\right] \\
\left.+d(\omega)\left|\left[G_{a}^{R}\right]^{-1}(-\omega)\right|^{2}\right) .
\end{array}
$$

This correlation function corresponds directly to the measurable power spectrum of the outcoupled cavity field. In Fig. 3, we plot the spectrum $C_{a}(\omega)$ for variable coupling $y$ from 0 to the critical point $y_{c}$ in the form of a color map. For better visibility, the bottom panel shows the spectrum at three different values of the coupling $y$, such that the functions correspond to the cross sections of the color map at zero coupling $y=0$, at an intermediate coupling $\left(y / y_{c}=0.5\right)$ and at a coupling in the critical domain $\left(y / y_{c}=0.99\right)$. For an uncoupled cavity mode $(y=0)$, the photon correlation function is a Lorentzian peak of width $2 \kappa$ centered at $\omega=\delta_{a}$. This peak is hardly visible in the colour code map in the top panel. The Lorentzian peak extends to negative frequencies, as the whole frequency range is shifted by the driving frequency in the rotating frame. The negative frequency part makes thus sense because of the external driving and it gives rise effectively to quantum noise which heats the system into a non-trivial steady state. For an intermediate coupling strength $\left(y=0.5 y_{c}\right)$, a doublet forms symmetrical to $\omega=0$ on the side of the Lorentzian peak. These two peaks correspond to the two poles $z_{1}=-z_{2}^{*}$ of the retarded Green's function on the negative complex half plane discussed in Subsection III B. This correspondence applies only away from the critical point, where the dynamics can still be well described by Lorentzian resonances associated with the poles. Nevertheless, the soft mode frequencies taken from Fig. 2, and plotted by a white solid line on top of the color map, fit accurately to the position of the peaks of the correlation function. Close to the critical point, in the interval where the real part of the soft-mode frequency vanishes, the doublet peaks merge into a large central peak around $\omega=0$ (solid red line in the bottom panel). In this range the use of the soft mode picture relying on Lorentzian resonances obviously breaks down. The criticality manifests itself in the divergence of this central peak as the coupling strength converges to $y_{c}$. In order to obtain the exponent, this divergence has to be considered and fitted by a power law.

\section{Atom correlation function}

The correlation function of the low frequency mode $b$ is derived from the atom-only action following a similar procedure as described in the previous subsection. The Keldysh component of the atom-only action reads

$$
\mathbf{D}_{b, 2 \times 2}^{K}=\left(\begin{array}{cc}
D(\omega)+g(\omega) & g(\omega) \\
g(\omega) & D(-\omega)+g(\omega)
\end{array}\right),
$$

with

$$
g(\omega)=-\frac{y^{2}}{4}\left[G_{a}^{K}(\omega)+G_{a}^{K}(-\omega)\right],
$$

where $G_{a}^{K}$ is the scalar Keldysh Green's function of mode $a$,

$$
G_{a}^{K}(\omega)=\frac{-2 i \kappa}{\left(\omega-\delta_{a}\right)^{2}+\kappa^{2}} .
$$

The retarded Green's function of the atom field is

$$
\begin{aligned}
\mathbf{G}_{b, 2 \times 2}^{R} & =\frac{1}{\operatorname{det}\left[\mathbf{G}_{b, 2 \times 2}^{R}\right]^{-1}} \\
& \left(\begin{array}{cc}
{\left[G_{b}^{A}\right]^{-1}(-\omega)+\Sigma_{b}^{R}(\omega)} & -\Sigma_{b}^{R}(\omega) \\
-\Sigma_{b}^{R}(\omega) & {\left[G_{b}^{R}\right]^{-1}(\omega)+\Sigma_{b}^{R}(\omega)}
\end{array}\right),
\end{aligned}
$$

with the self energy

$$
\Sigma_{b}^{R}(\omega)=-\frac{y^{2}}{2} \frac{\delta_{a}}{(\omega+i \kappa)^{2}-\delta_{a}^{2}} .
$$



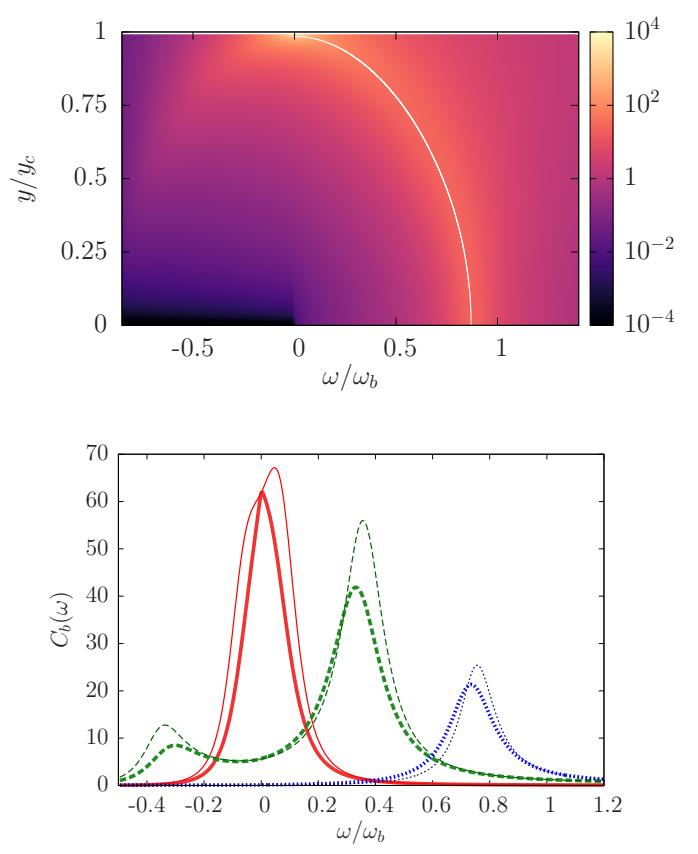

FIG. 4: (Color online) Correlation function of mode $b$ for various coupling strengths approaching the critical point. The top panel presents a color map of the correlation function in frequency space (x-axis) as a function of the relative coupling strenth (y-axis) for $s=0.8$. The white line shows the mode softening $\operatorname{Re} \omega_{s}$ shown in Fig. 2. In the bottom panel, we compare two complementer bath exponents $s=0.8$ (thick lines) and $s=1.8$ (thin lines) for couplings $y=0$ (dotted blue), 0.5 (dashed green) and 0.99 (solid red). Other parameters are the same as in Fig. 3.

The advanced Green's function is calculated from the symmetry $\mathbf{G}_{b, 2 \times 2}^{A}=\left[\mathbf{G}_{b, 2 \times 2}^{R}\right]^{\dagger}$, and $\mathbf{D}_{2 b, \times 2}^{K}$ is given by Eq. (41).

The interesting part of the Keldysh Green's function is its first diagonal element which gives the Fourier transform of the atom correlation function $C_{b}(t)=$ $\left\langle\left\{b(t), b^{\dagger}(0)\right\}\right\rangle$, that is $C_{b}(\omega)=\left[\mathbf{G}_{b, 2 \times 2}^{K}\right]_{11}$,

$$
\begin{aligned}
C_{b}(\omega)= & \frac{-i}{\left|\operatorname{det}\left[\mathbf{G}_{b, 2 \times 2}^{R}\right]^{-1}\right|^{2}}\left(D ( \omega ) \left[\left|\left[G_{b}^{R}\right]^{-1}(-\omega)\right|^{2}\right.\right. \\
+ & \left.2 \operatorname{Re}\left\{\Sigma_{b}^{R}(\omega)\left[G_{b}^{R}\right]^{-1}(-\omega)\right\}+\left|\Sigma^{R}(\omega)\right|^{2}\right] \\
& \left.D(-\omega)\left|\Sigma^{R}(\omega)\right|^{2}+d(\omega)\left|\left[G_{b}^{R}\right]^{-1}(-\omega)\right|^{2}\right) .
\end{aligned}
$$

The top panel of Fig. 4 explores how the correlation function of mode $b$ changes when $y$ approaches $y_{c}$. Upon the color map we plot the real part of the soft mode pole (shown in Fig. 2). Although the pole gives the position of the resonance quite accurately, we stress that these peaks are not Lorenzian due to the frequency-dependent numerator of the correlation function, Eq. (46). As increasing the coupling, the single non-Lorentzian peak for $y=0$ moves towards zero, and a second peak appears on the negative frequency side due to the interaction with the

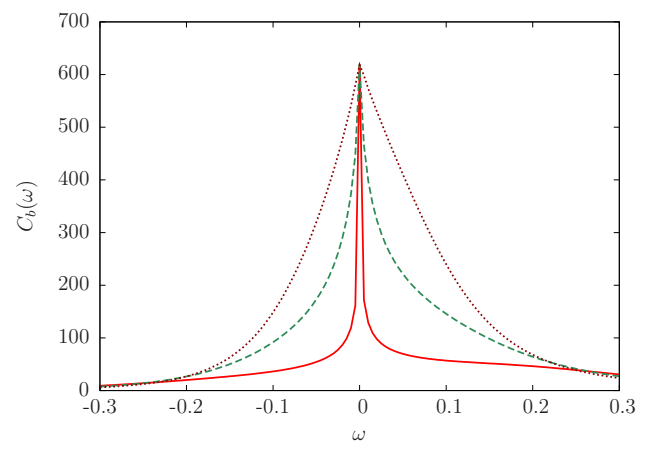

FIG. 5: (Color online) Atomic correlation functions close to the critical point for $y=0.99 y_{c}$. The width of the peak decreases with the parameter $s$, that is $s=0.7$ (dotted brown), $s=0.5$ (dashed green) and $s=0.3$ (solid red). Other parameters are the same as in Fig. 6.

cavity photons. Approaching the critical point, the two peaks merge to form a diverging peak at zero frequency. In the bottom panel of Fig. 4, we plot two sets of correlation functions, for $s=0.8$ (thick lines) and $s=1.8$ (thin lines), in order to demonstrate the quasi-periodicity of the correlation function with respect to the bath exponent, explained earlier in connection with Eq. (9). The trigonometric factor in the expression (20) of $K^{R}(\omega>0)$ is periodic, and remains the same for $s \rightarrow s+1$, only the exponent of $\omega$ is different in the two cases. We observe that the central peak is sharper for the smaller $s$ value. The correlation functions give the same value at $\omega=0$ for the two different exponents $s$, since the mode density of the colored bath vanishes anyway at zero frequency.

\section{Critical exponent}

As we indicated before, the critical exponent can be calculated only directly from the diverging fluctuations by determining the power law exponent of the divergence. The excitation numbers of mode $a$ and $b$ in the steady state are the equal-time correlations, i.e. $\left\langle a^{\dagger}(0) a(0)\right\rangle=$ $\left(C_{a}(t=0)-1\right) / 2$, which can be calculated by integrating the correlation functions in Fourier space

$$
C_{a, b}(t=0)=\int \frac{\mathrm{d} \omega}{2 \pi} C_{a, b}(\omega) .
$$

Let us investigate the atomic correlation function $C_{b}(\omega)$ at a coupling very close to the critical point $\left(y=0.99 y_{c}\right)$, which is shown in Figure 5 for three different values of the exponent $s$. These spectra are manifestly far from a Lorentzian form, indicating that the soft mode picture cannot account for the divergence of the fluctuations. The peak points match at zero frequency, at the same time, the smaller the exponent $s$, the narrower the peak. Hence, the area below the peaks, giving the integral in Eq. (47), varies as a function of the exponent $s$. 


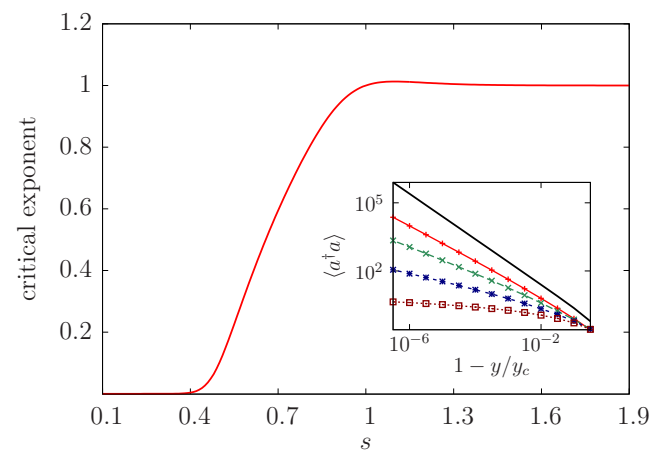

FIG. 6: (Color online) Critical exponent as a function of the bath exponent $s$ of the colored reservoir. The inset shows the power-law scaling of the photon number. Parameters: $\delta_{a}=2 \omega_{b}, \kappa=0.5 \omega_{b}, \gamma=0.1 \omega_{b}, \mu=0$.

The exponent is extracted from a power-law fit on the excitation numbers plotted against $1-y / y_{c}$ close to the critical point $\left|y-y_{c}\right|<10^{-4}$. In the inset of Fig. 6, we show the scaling of the photon number for a set of values of the parameter $s$. Even in the presence of the colored noise, one clearly recognizes the power-law divergence in the close vicinity of the critical point.

The main panel of Fig. 6 presents the central result of our paper, where we explore how the critical exponent of the system varies as a function of the bath exponent $s$. For $s>1$, we recover the exponent 1 , that is the original open-system exponent of the Dicke model [32]. This thermal-like exponent arises due to the Markovian dissipation process, where the fluctuations are Gaussian, similarly to thermal fluctuations. We find that a superOhmic reservoir does not affect this critical exponent. In contrast, the critical fluctuations are significantly reshaped in the sub-Ohmic case for $s<1$, where we find a monotonic decrease of the critical exponent with decreasing bath parameter $s$. Moreover, for $s<0.4$, the critical fluctuations are completely suppressed by the colored reservoir, and $\left\langle a^{\dagger} a\right\rangle$ and $\left\langle b^{\dagger} b\right\rangle$ remain finite at the critical point. This means that the driving and the photon loss induced noise of the cavity mode is effectively dissipated into the colored reservoir.

\section{THERMAL EFFECTS}

There has been a discussion whether the phase transition can be considered a classical thermal one in the open-system Dicke model. Although the cavity fluctuations are Gaussian, similarly to thermal fluctuations, certain properties of the critical behaviour make this identification doubtful. In particular, the cavity and atomic subsystems have different effective temperatures in the steady-state, hence the driven system as a whole cannot be considered to be in a thermal equilibrium [33].

Within the Keldysh formalism, we can consider the thermal effects when the low-frequency colored bath has a finite temperature. For instance, in the experimental realization, the thermal fraction of the BEC corresponds to the thermal occupation of the phonon modes that form the colored reservoir. In our general theory, we include the temperature in the inverse Keldysh component of mode $b$, Eq. (7b). Meanwhile, we keep the Green's functions of mode $a$ unchanged, because the temperature is negligible at the pump frequency $\hbar \omega_{p} \gg k_{B} T$. It is only relevant for the critical dynamics at low frequencies.

\section{A. Thermal equilibrium of mode $b$}

Before turning to the coupled system, we investigate the thermal average of the population in mode $b$ coupled to a finite-temperature colored bath. The temperature of the reservoir is included via the Keldysh component Eq. (7b) in the distribution function $F(\omega)=$ $\operatorname{coth}\left\{\hbar \omega / k_{B} T\right\}$. The thermal occupation of oscillator $b$ is calculated as previously described. When simplifying the correlation function Eq. (46) for $y=0$, we obtain the following integral for the zero-time correlation function (according to Eq. (47)),

$$
C_{b}(t=0)=\int d \omega \frac{\rho(\omega) \operatorname{coth}\left(\hbar \omega / 2 k_{B} T\right)}{\left(\omega-\omega_{b}-\operatorname{Re} K(\omega)\right)^{2}+(\operatorname{Im} K(\omega))^{2}} .
$$

In order to get the usual thermal occupation number of the oscillator, one has to assume that the denominator of the integrand has a sharp peak, so that the cotangent hyperbolic function can be taken out of the integral. Accordingly, it should be a flat function around the resonance $\omega_{r}$, given by $\omega_{r}-\omega_{b}-\operatorname{Re} K\left(\omega_{r}\right)=0$, that leads to the condition $\left.\left.\mid \gamma_{r} \operatorname{coth}^{\prime}\left(\hbar \omega_{r} / 2 k_{B} T\right)\right) \mid \ll \operatorname{coth}\left(\hbar \omega_{r} / 2 k_{B} T\right)\right)$ with $\gamma_{r}=\operatorname{Im} K\left(\omega_{r}\right)$. Also, the coupling-density profile has to be a flat function of $\omega$ at resonance, $\left|\gamma_{r} \rho^{\prime}\left(\omega_{r}\right)\right| \ll$ $\rho\left(\omega_{r}\right)$. With these approximations one obtains the expected thermal occupation of the oscillator

$$
C_{b}(t=0) \stackrel{\gamma_{r} \rightarrow 0}{\longrightarrow} \operatorname{coth}\left(\hbar \tilde{\omega}_{b} / 2 k_{B} T\right),
$$

with the renormalized oscillator frequency given by the resonance $\tilde{\omega}_{b}=\omega_{r}$. The limit $\gamma_{r} \rightarrow 0$ ensures that the remaining integral

$$
\int d \omega \frac{\rho(\omega)}{\left(\omega-\omega_{b}-\operatorname{Re} K(\omega)\right)^{2}+(\operatorname{Im} K(\omega))^{2}} \longrightarrow 1
$$

In Fig. 7 , we compare the excitation numbers in mode $b$ calculated numerically from the integral Eq. (48) to the thermal equilibrium value, Eq. (49) without the frequency renormalisation, i.e., $\tilde{\omega}_{b} \simeq \omega_{b}$ (solid red line). When the coupling to the reservoir is large $\gamma=0.5 \omega_{b}$ (blue crosses) the excitation number is noticabley above the thermal occupation, while for small reservoir coupling $\left(\gamma=0.03 \omega_{b}\right.$, green circles) the expected analytical curve of thermal equilibrium is well reproduced.

Note that, although the Keldysh path integral approach is not perturbative in $\gamma$ when we integrate out 


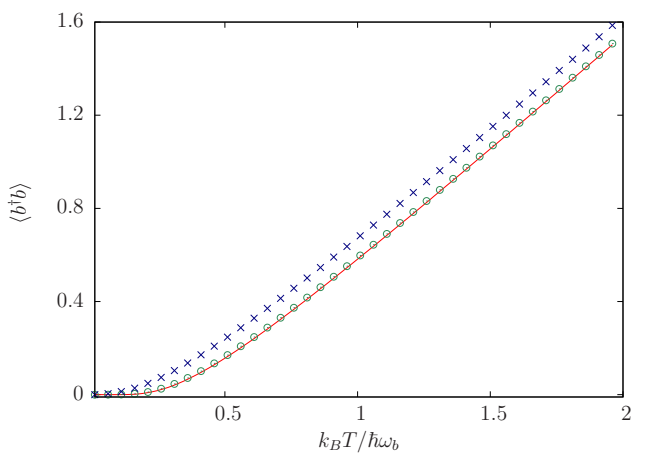

FIG. 7: (Color online) Thermal occupation of mode $b$ calculated from the correlation function at $y=0$ as a function of the bath temperature. The analytical curve depicts the thermal state (solid red line), which is reached for small reservoir coupling $\gamma=0.03 \omega_{b}$ (green circles). For $\gamma=0.5 \omega_{b}$ (blue crosses), higher occupation numbers are obtained for the colored reservoir. Parameters: $s=0.6$ and $\mu=0$.

the reservoir modes, in the end, we still need to consider small coupling to the reservoir so as to fulfill the thermodynamics of the oscillator. This is related to the initial assumption that the bath is in thermal equilibrium. Once the system is strongly coupled to the reservoir, the system back-action significantly changes the dynamics of the reservoir modes and the thermal equilibrium is lost. Normally, realistic decay rates are orders of magnitude smaller than the oscillator frequency $\left(\gamma \ll \omega_{b}\right)$. The numerical calculations, however, are easier to carry out for larger decay rates, and this will be our choice.

\section{B. Thermal cross-excitation effects}

In Figure 8 the cavity photon number is plotted as a function of the relative coupling $y / y_{c}$ for different temperatures of the colored bath (top panel). The thermal excitation of mode $b$ gives rise to an increase of the cavity photon number through photon scattering. This crosseffect reflects again the driving as a substantial feature of the system. High-frequency photons can be created from the low-frequency thermal excitations. The photon number curve is pushed upward from the $T=0$ curve (solid red); the larger the $T$, the larger the shift.

Similar behaviour can be seen on the atomic excitation number (bottom panel) for low $T<1$. The interesting new feature takes place at higher temperature, $k_{B} T=10 \hbar \omega_{b}$ : the population in mode $b$ decreases with the atom-cavity coupling before the diverging peak at the critical point. This effect can be attributed to the cavity cooling mechanism. The cavity dissipation channel through the atom-photon coupling can extract energy from the atomic mode. Then, the new excitation number is the result of the competition between the cavity cooling and thermalization. Here we stress that the theory is valid for small system-bath coupling, which ensures that the bath can remain in thermal equilibrium.
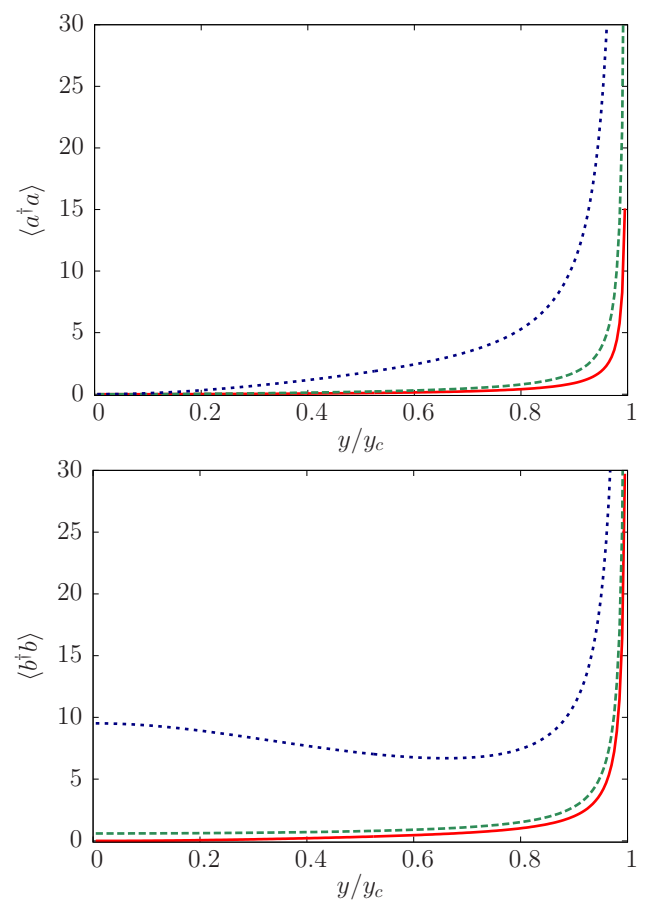

FIG. 8: (Color online) Excitation number in the cavity mode $a$ (top panel) and the atomic mode $b$ (bottom panel) as a function of the relative coupling strength $y / y_{c}$ at different temperatures $T=0,1$, and $10 \hbar \omega_{b} / k_{B}$. Parameters: $\delta_{a}=2$, $\kappa=2, g=0.1 \omega_{b}$.

\section{Critical exponent}

Finally, we study the role of temperature on the criticality of the driven-damped system. We wish to explore how the critical exponent changes with the bath exponent when the colored reservoir has a finite temperature $T=\hbar \omega_{b} / k_{B}$. In this analysis, the chemical potential of the colored reservoir, introduced in Eq. (7b), plays a significant role below $s=1.3$. Deeply in the super-Ohmic regime $s>1.3$, the same zero-temperature exponent 1 is obtained for $\mu=0$. For $s<1.3$, our calculation breaks down indicating that the Fourier transform of the correlation function is divergent. Finite chemical potential has to be introduced in order to account for the state of the low-frequency reservoir modes, i.e., in order to regularize the diverging particle number in the reservoir modes around $\omega=0$. We performed the calculation on the diverging population in the modes at several chemical potential choices. Figure 9 shows that the overall behaviour of the critical exponent as a function of the exponent of the reservoir spectral density function is the same as at $T=0$. The overall behaviour of the plotted curves is also unaffected by the chemical potential. For larger chemical potential (dotted brown), the zerotemperature result (solid red) is obtained. For smaller chemical potential, the central part of the curve is shifted a bit more towards lower critical exponents, all this happening in the interval $0.4<s<1.3$. In the super-Ohmic 


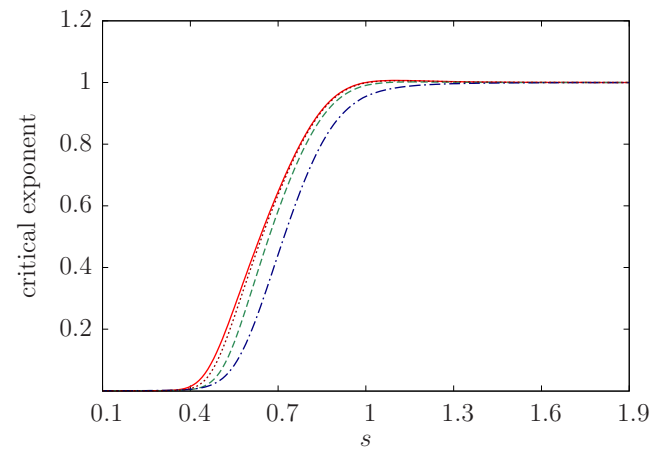

FIG. 9: (Color online) Critical exponent as a function of the bath exponent $s$ of the colored reservoir for different chemical potentials $\mu=-0.1$ (dotted brown), -0.01 (dashed green) and $-0.001 \omega_{b}$ (dashed-dotted blue). Other parameters are $\kappa=0.5 \omega_{b}, \gamma=0.1 \omega_{b}, T=1 \hbar \omega_{b} / k_{B}$. We plot the zero temperature curve (solid red) of Fig. 6 for reference.

regime (above $s>1.3$ ), the cavity dissipation dominates the critical exponent, and the colored bath proves to be irrelevant regarding the criticality.

\section{CONCLUSION AND OUTLOOK}

In this paper we studied the effects of a colored reservoir on the criticality occurring in a driven-dissipative system. Our model corresponds to the cavity QED realization of the Dicke model, where a high-frequency driven cavity mode is coupled to a low-frequency condensate ex- citation mode. We demonstrated that the critical exponent of the superradiant phase transition is highly sensitive to the spectral features of the low-frequency reservoir. In particular, we found that the critical exponent varies monotonously as a function of the bath exponent in the domain of sub-Ohmic reservoirs. The criticality vanishes completely at a given value of the bath exponent. For super-Ohmic reservoirs, the critical exponent is determined by the Markovian loss channel and is constant 1 . We showed that the non-vanishing temperature does not break this dependence on the spectral density function of the bath, and non-trivial exponents are obtained for sub-Ohmic reservoirs.

Besides the field of cavity QED there are other systems whose driven-dissipative nature is inherent to their physical realization. For instance, Dicke-type systems can be designed in circuit QED [46-48], where nonGaussian noise appears from the motion of electrons [49]. Other examples for out-of-equilibrium systems are polariton Bose-Einstein condensates created in semiconductor heterostructures, where dissipation is intrinsic to the dynamics $[50,51]$.

\section{Acknowledgments}

The authors thank fruitful discussions with Z. Kurucz and G. Kónya. This work was supported by the Hungarian Academy of Sciences (Lendület Program, LP2011016) and the National Research, Development and Innovation Office (K115624). D. N. acknowledges support from the János Bolyai Research Scholarship of the Hungarian Academy of Sciences.
[1] E. G. Dalla Torre, E. Demler, T. Giamarchi, and E. Altman, Nature Physics 6, 806 (2010).

[2] S. Diehl, A. Tomadin, A. Micheli, R. Fazio, and P. Zoller, Phys. Rev. Lett. 105, 015702 (2010).

[3] E. G. Dalla Torre, E. Demler, T. Giamarchi, and E. Altman, Phys. Rev. B 85, 184302 (2012).

[4] R. Chitra and O. Zilberberg, Phys. Rev. A 92, 023815 (2015).

[5] M. Schiró, C. Joshi, M. Bordyuh, R. Fazio, J. Keeling, and H. E. Türeci, Phys. Rev. Lett. 116, 143603 (2016).

[6] L. M. Sieberer, S. D. Huber, E. Altman, and S. Diehl, Phys. Rev. Lett. 110, 195301 (2013).

[7] J. Marino and S. Diehl, Phys. Rev. Lett. 116, 070407 (2016).

[8] J. Marino and S. Diehl, Phys. Rev. B 94, 085150 (2016).

[9] J. Lang and F. Piazza, Phys. Rev. A 94, 033628 (2016).

[10] A. Le Boité, G. Orso, and C. Ciuti, Phys. Rev. Lett. 110, 233601 (2013).

[11] A. Le Boité, G. Orso, and C. Ciuti, Phys. Rev. A 90, 063821 (2014).

[12] T. Griesser and H. Ritsch, Phys. Rev. Lett. 111, 055702 (2013).

[13] S. Ostermann, F. Piazza, and H. Ritsch, Phys. Rev. X 6, 021026 (2016).

[14] S. Sachdev, Quantum Phase Transitions (Cambridge
University Press, 2011), ISBN 978-0-521-51468-2.

[15] P. Strack and S. Sachdev, Phys. Rev. Lett. 107, 277202 (2011).

[16] F. Piazza and P. Strack, Phys. Rev. Lett. 112, 143003 (2014).

[17] M. J. Hwang, R. Puebla, and M. B. Plenio, Phys. Rev. Lett. 115, 180404 (2015).

[18] A. E. Niederle, G. Morigi, and H. Rieger, Phys. Rev. A 94, 033607 (2016).

[19] K. Baumann, C. Guerlin, F. Brennecke, and T. Esslinger, Nature 464, 1301 (2010).

[20] K. Baumann, R. Mottl, F. Brennecke, and T. Esslinger, Phys. Rev. Lett. 107, 140402 (2011).

[21] R. Mottl, F. Brennecke, K. Baumann, R. Landig, T. Donner, and T. Esslinger, Science 336, 1570 (2012).

[22] D. Schmidt, H. Tomczyk, S. Slama, and C. Zimmermann, Phys. Rev. Lett. 112, 115302 (2014).

[23] M. P. Baden, K. J. Arnold, A. L. Grimsmo, S. Parkins, and M. D. Barrett, Phys. Rev. Lett. 113, 020408 (2014).

[24] J. Klinder, H. Keßler, M. Wolke, L. Mathey, and A. Hemmerich, P. Natl. Acad. Sci. USA p. 201417132 (2015).

[25] J. Klinder, H. Kessler, M. R. Bakhtiari, M. Thorwart, and A. Hemmerich, Phys. Rev. Lett. 115, 230403 (2015).

[26] R. Landig, L. Hruby, N. Dogra, M. Landini, R. Mottl, T. Donner, and T. Esslinger, Nature 532, 476 (2016). 
[27] A. J. Kollár, A. T. Papageorge, K. Baumann, M. A. Armen, and B. L. Lev, New Journal of Physics 17, 043012 (2015).

[28] A. J. Kollár, A. T. Papageorge, V. D. Vaidya, Y. Guo, J. Keeling, and B. L. Lev, arXiv p. 1606.04127 (2016).

[29] F. Brennecke, R. Mottl, K. Baumann, R. Landig, T. Donner, and T. Esslinger, P. Natl. Acad. Sci. USA 110, 11763 (2013).

[30] J. Vidal and S. Dusuel, Europhys. Lett. p. 817 (2007).

[31] D. Nagy, G. Kónya, G. Szirmai, and P. Domokos, Phys. Rev. Lett. 104, 130401 (2010).

[32] D. Nagy, G. Szirmai, and P. Domokos, Phys. Rev. A 84, 043637 (2011).

[33] E. G. Torre, S. Diehl, M. D. Lukin, S. Sachdev, and P. Strack, Phys. Rev. A 87, 023831 (2013).

[34] D. Nagy and P. Domokos, Phys. Rev. Lett. 115, 043601 (2015).

[35] G. Kónya, G. Szirmai, D. Nagy, and P. Domokos, Phys. Rev. A 89, 051601 (2014).

[36] G. Kónya, G. Szirmai, and P. Domokos, Phys. Rev. A 90, 013623 (2014).

[37] H. Ritsch, P. Domokos, F. Brennecke, and T. Esslinger, Rev. Mod. Phys. 85, 553 (2013).

[38] B. M. Garraway, Philosophical Transactions of the Royal Society of London A: Mathematical, Physical and Engineering Sciences 369, 1137 (2011).

[39] J. Cardy, Scaling and Renormalization in Statistical
Physics (Cambridge University Press, 1996).

[40] C. Emary and T. Brandes, Phys. Rev. E 67, 066203 (2003).

[41] Z. Kurucz and K. Mølmer, Phys. Rev. A 81, 032314 (2010).

[42] C. Cohen-Tannoudji, J. Dupont-Roc, and G. Grinberg, Atom-Photon Interactions (Wiley, New York, 1992).

[43] A. J. Leggett, S. Chakravarty, A. T. Dorsey, M. P. A. Fisher, A. Garg, and W. Zwerger, Rev. Mod. Phys. 59, 1 (1987).

[44] E. M. Kessler, G. Giedke, A. Imamoglu, S. F. Yelin, M. D. Lukin, and J. I. Cirac, Phys. Rev. A 86, 012116 (2012).

[45] H. Eleuch and I. Rotter, Phys. Rev. E 87 (2013).

[46] P. Nataf, A. Baksic, and C. Ciuti, Phys. Rev. A 86, 013832 (2012).

[47] A. Baksic and C. Ciuti, Phys. Rev. Lett. 112, 173601 (2014).

[48] J. Lolli, A. Baksic, D. Nagy, V. E. Manucharyan, and C. Ciuti, Phys. Rev. Lett. 114, 183601 (2015).

[49] E. Paladino, Y. . M. Galperin, G. Falci, and B. . L. Altshuler, Rev. Mod. Phys. 86, 361 (2014).

[50] I. Carusotto and C. Ciuti, Rev. Mod. Phys. 85, 299 (2013).

[51] G. Wachtel, L. M. Sieberer, S. Diehl, and E. Altman, Phys. Rev. B 94, 104520 (2016). 